\title{
Número de folhas e comprimento de estaca no enraizamento de sanquésia
}

\author{
Number of leaves and cutting length in the rooting of sanchesia \\ Número de hojas y longitud de corte en el enraizamiento de sanchesia
}

Carla Marins Santos Santana Viana

ORCID: https://orcid.org/0000-0003-2097-4462

Universidade Tecnológica Federal do Paraná, Brasil

E-mail: santosclarice323@gmail.com

Michel Anderson Masiero

ORCID: https://orcid.org/0000-0003-2242-515X

Universidade Estadual do Oeste do Paraná, Brasil

E-mail: michel_masiero2@hotmail.com

Elizabete Artus Berte

ORCID: https://orcid.org/0000-0002-3133-894X

Universidade Tecnológica Federal do Paraná, Brasil

E-mail: elizabeteberte9@gmail.com

Silvane Zancanaro de Oliveira

ORCID: https://orcid.org/0000-0003-4546-7854

Universidade Tecnológica Federal do Paraná, Brasil

E-mail: silvanezanoli@gmail.com

Erivelto Folhato Tolfo

ORCID: https://orcid.org/0000-0001-6835-775X

Universidade Tecnológica Federal do Paraná, Brasil

E-mail: eriveltoftolfo@gmail.com

Roberta Aparecida Folhiato

ORCID: https://orcid.org/0000-0003-3382-1561

Universidade Tecnológica Federal do Paraná, Brasil

E-mail: folhiato.roberta@gmail.com

Edineia Kaiper

ORCID: https://orcid.org/0000-0003-0055-3150

Universidade Tecnológica Federal do Paraná, Brasil

E-mail: edineiakaiper@gmail.com

Vilmar Acorde de Souza

ORCID: https://orcid.org/0000-0002-6592-9465

Universidade Tecnológica Federal do Paraná, Brasil

E-mail: vilmaracorde@gmail.com

Ana Leticia de Campos

ORCID: https://orcid.org/0000-0002-9905-146X

Universidade Tecnológica Federal do Paraná, Brasil

E-mail: analeticiacampos95@hotmail.com

Daniela Macedo de Lima

ORCID: https://orcid.org/0000-0002-8018-3163

Universidade Tecnológica Federal do Paraná, Brasil

E-mail: danielamlima@utfpr.edu.br

\begin{abstract}
Resumo
Sanchezia oblonga Ruiz \& Pav. é uma espécie ornamental e florística, muito utilizada no paisagismo e em jardins domésticos, atuando como uma das principais fontes alimentícias para a manutenção de polinizadores durante a estação do inverno. O objetivo deste trabalho foi avaliar a influência de folhas e o tamanho das estacas no enraizamento de sanquésia. Este trabalho foi constituído por dois experimentos, ambos, foram conduzidos em casa de sombra no Viveiro Florestal da Universidade Tecnológica Federal do Paraná - Câmpus Dois Vizinhos (UTFPR-DV). O delineamento experimental foi inteiramente casualizado (DIC), com três tratamentos, quatro repetições de 10 estacas por parcela. O primeiro experimento consistiu na confecção de estacas de $6 \mathrm{~cm}$ de comprimento, sendo os tratamentos constituídos por estacas sem folhas, com um par de folhas e com dois pares de folhas reduzidos à metade. No segundo, avaliou-se estacas com três comprimentos diferentes $(6,12$ e $18 \mathrm{~cm})$ e com um par de folhas reduzido à metade. Em ambos experimentos, as estacas foram plantadas em tubetes de $120 \mathrm{~cm}^{3}$ contendo o substrato vermiculita na granulometria fina. Após 73 dias, foram analisadas as porcentagens de enraizamento, sobrevivência, mortalidade, brotações, número e comprimento médio de raízes por estaca e número de brotações. Em relação aos tamanhos das estacas não houve diferença significativa, no entanto, constatou-se que a espécie é facilmente propagada pela técnica de estaquia, visto que a média geral de enraizamento foi de $98,86 \%$. O enraizamento das estacas com folhas foi
\end{abstract}


superior quando comparado às sem folhas, com isso, recomenda-se o uso de estacas com um ou dois pares de folhas reduzidas à metade folha para produção de mudas desta espécie.

Palavras-chave: Sanchezia oblonga; Estaquia; Propagação-vegetativa.

\begin{abstract}
Sanchezia oblonga Ruiz \& Pav. is an ornamental and floristic species, widely used in landscaping and domestic gardens, acting as one of the main food sources for the maintenance of pollinators during the winter season. The objective of this work was to evaluate the influence of leaves and the size of cuttings on the rooting of sanchesia. This work consisted of two experiments, both of which were conducted in a shade house at the Forest Nursery of the Federal Technological University of Paraná - Câmpus Dois Vizinhos (UTFPR-DV). The experimental design was completely randomized (DIC), with three treatments, four replications of 10 cuttings per plot. The first experiment consisted of making cuttings that were standardized at $6 \mathrm{~cm}$ in length, and the treatments consisted of cuttings without leaves, with a pair of leaves cut in half and cuttings with two pairs of leaves cut in half. In the second, cuttings with three different lengths $(6,12$ and $18 \mathrm{~cm})$ were evaluated and with a pair of leaves cut in half. In both experiments, the cuttings were planted in $120 \mathrm{~cm}^{3}$ tubes containing the vermiculite substrate in fine granulometry. After 73 days, rooting percentages, survival, mortality, shoots, number and average length of roots per cut and number of shoots were analyzed. Regarding the size of the cuttings, there was no significant difference, however, it was found that the species is easily propagated by the cutting technique, since the general rooting average was $98.86 \%$. The rooting of cuttings with leaves was superior when compared to cuttings without leaves, therefore, it is recommended to use cuttings with one or two pairs of leaves reduced to half leaf for the production of seedlings of this species.
\end{abstract}

Keywords: Sanchezia oblonga; Cutting; Vegetative-propagation.

\title{
Resumen
}

Sanchezia oblonga Ruiz \& Pav. es una especie ornamental y florística, muy utilizada en jardinería y jardines domésticos, actuando como una de las principales fuentes de alimento para el mantenimiento de los polinizadores durante la temporada invernal. El objetivo de este trabajo fue evaluar la influencia de las hojas y el tamaño de los esquejes en el enraizamiento de la sanchesia. Este trabajo consistió en dos experimentos, ambos realizados en una casa de sombra en el Vivero Forestal de la Universidad Tecnológica Federal de Paraná - Câmpus Dois Vizinhos (UTFPR-DV). El diseño experimental fue completamente al azar (DIC), con tres tratamientos, cuatro repeticiones de 10 esquejes por parcela. El primer experimento consistió en realizar esquejes de $6 \mathrm{~cm}$ de largo, con tratamientos que consistieron en esquejes sin hojas, con un par de hojas y con dos pares de hojas cortadas por la mitad. En el segundo, se evaluaron esquejes con tres longitudes diferentes $(6,12$ y $18 \mathrm{~cm})$ y con un par de hojas cortadas por la mitad. En ambos experimentos, los esquejes se plantaron en tubos de $120 \mathrm{~cm}^{3}$ que contenían el sustrato de vermiculita en granulometría fina. A los 73 días se analizaron los porcentajes de enraizamiento, supervivencia, mortalidad, brotes, número y longitud promedio de raíces por corte y número de brotes. En cuanto a los tamaños de estacas, no hubo diferencia significativa, sin embargo, se encontró que la especie se propaga fácilmente por la técnica de corte, ya que el promedio general de enraizamiento fue de 98,86\%. El enraizamiento de los esquejes con hojas fue superior en comparación con los esquejes sin hojas, por lo que se recomienda utilizar esquejes con uno o dos pares de hojas reducidos a media hoja para la producción de plántulas de esta especie.

Palabras clave: Sanchezia oblonga; Corte; Propagación-vegetativa.

\section{Introdução}

Sanchezia oblonga Ruiz \& Pav. (Sanquésia, Acanthaceae) é uma espécie natural da América do Sul, mais especificamente do Peru e Equador, se desenvolvendo em variados climas como o tropical e subtropical. Apresenta caráter arbustivo, copa arredondada e frondosa, podendo atingir até 3 metros de altura. Possui folhas grandes, lanceoladas, glabras, opostas, de coloração esverdeada, também apresenta nervuras em baixo relevo e amareladas, além de inflorescências formadas por brácteas vermelhas-alaranjadas e pequenas flores amareladas, tubulares, bem atrativas para os polinizadores (Lorenzi; Souza, 2008).

A espécie é muito recomendada para utilização em parques e jardins, tanto pela produção de beleza cênica, quanto pela manutenção da fauna, principalmente para diferentes grupos de polinizadores. Suas flores são amplamente visitadas por espécies coletoras de pólen e néctares, como beija-flores, borboletas e abelhas (Lorenzi; Souza, 2008). É importante salientar que sua fase de reprodução floral ocorre durante o outono e o inverno, em épocas que a produção florística de outras espécies vegetais está mais escassa (Espindola; Orenha, 2007; Weiss, et al., 2018).

As formas de reprodução de uma planta são caracterizadas como sexuada (via semente) e assexuada (via propágulo), 
sendo a última realizada a partir de partes vegetais com potencial de desenvolver raízes adventícias (Xavier; Wendling; Silva, 2013; Hartmann, et al., 2018). S. oblonga é exclusivamente propagada pela técnica de estaquia, principalmente pelo seu elevado potencial de enraizamento (Lorenzi; Souza, 2008; Weiss, et al., 2018). As principais vantagens da propagação vegetativa consistem na produção de mudas homogêneas, com características idênticas a planta matriz e a antecipação da produção floral (Fachinello, et al., 2005; Marchi, et al., 2018).

Alguns fatores internos e externos são determinantes para o sucesso do enraizamento (SOUZA et al., 2015). Dentre os endógenos estão a composição química das plantas, as fontes de carboidratos, proteínas, vitaminas, aminoácidos, compostos fenólicos, hormônios vegetais dentre outros), a idade da planta, a presença de folhas e gemas, etc. Quanto aos fatores ambientais podem ser citados a umidade, luminosidade e temperatura (Xavier; Wendling; Silva, 2013; Hartmann, et al., 2018).

A presença de folhas na estaca está diretamente ligada a formação de uma nova estrutura radicular, tendo em vista a importância das folhas para produção de assimilados no processo de fotossíntese (Costa, et al., 2016; Taiz, et al., 2017; Masiero, et al., 2021). Contudo, a área foliar em excesso pode causar danos ao enraizamento, devido à desidratação do ramo causado pela transpiração das folhas (Hartmann, et al., 2018). O tamanho da estaca impacta diretamente na condição de reservas nutricionais, pois, geralmente estacas de maior comprimento armazenam maiores quantidades de reservas e gemas (Pizzatto, et al., 2011), sendo muito importantes para a formação de raízes adventícias.

Este trabalho teve como objetivo estudar a influência de folhas e o comprimento das estacas no enraizamento de sanquésia.

\section{Metodologia}

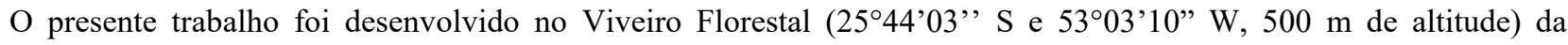
Universidade Tecnológica Federal do Paraná - Câmpus Dois Vizinhos (UTFPR-DV). Segundo classificação de Köppen, o clima da região é subtropical, do tipo Cfa, com temperatura do mês mais frio entre $-3{ }^{\circ} \mathrm{C}$ a $18{ }^{\circ} \mathrm{C}$, sempre úmido, chuva em todos os meses do ano e temperatura do mês mais quente superior a $22{ }^{\circ} \mathrm{C}$, mas no mínimo quatro meses com temperatura maior que $10^{\circ} \mathrm{C}$ (Alvares, et al., 2013).

Este estudo é de caráter quantitativo seguindo padrões metodológicos de Pereira, et al. (2018), esse estudo e resultado de dois experimentos realizados em casa de sombra simultaneamente com a espécie $S$. oblonga, ambos no período de agosto a novembro de 2016, composto por dois tipos de testes de estaquia. A coleta de ramos semilenhosos foi realizada no período da manhã, de uma planta matriz situada nos jardins do próprio Câmpus.

Durante a confecção das estacas de ambos os experimentos, as mesmas foram colocadas em bandejas com água, para evitar a desidratação até o momento do plantio. O estaqueamento foi realizado em tubetes de polietileno de $120 \mathrm{~cm}^{3}$ contendo substrato vermiculita de granulometria fina umedecido previamente. Em seguida foram levadas a casa de sombra de coloração preta e sombreamento $50 \%$ de transparência, com temperatura e umidade relativa do ar (UR\%) ambiente. A temperatura média e (UR\%) ficaram respectivamente: agosto $\left(15,8^{\circ} \mathrm{C} ; 76 \%\right)$, setembro $\left(18,7^{\circ} \mathrm{C} ; 62 \%\right)$, outubro $\left(20,3^{\circ} \mathrm{C} ; 73 \%\right)$ e novembro $\left(22,1^{\circ} \mathrm{C} ; 66 \%\right)$ (INMTE, 2016). As irrigações foram diárias e manuais em 3 períodos nos turnos da manhã e tarde, com duração aproximada de 20 minutos.

O delineamento experimental foi inteiramente casualizado (DIC) para ambos os estudos, onde cada um possuía três tratamentos (EXP 1: sem; 1 par e 2 pares de folhas e EXP 2: 6; 12 e $18 \mathrm{~cm}$ ), contendo quatro repetiç̃os cada e 10 estacas por repetição.

Após 73 dias de ambos experimentos, foram avaliadas as variáveis porcentagem de enraizamento (E), mortalidade (M), brotações (B) e sobrevivência (S), bem como número de raízes (NR), comprimento médio das três maiores raízes por estaca (CMTR) e número de brotações (NB). A sobrevivência foi analisada somente no EXP 1 (ausência e presença de folhas). 
Os dados foram submetidos à análise de variância e ao teste de Tukey $(\mathrm{P}<0,05)$, ambos a $5 \%$ de probabilidade de erro. Foi utilizando como auxilio o software SISVAR 5,3 (Ferreira, 2011).

\section{Resultados e Discussão}

\section{Experimento 1}

Em relação ao primeiro estudo avaliando a influência das folhas no enraizamento de sanquésia, houve diferença significativa para as variáveis: porcentagem de enraizamento (E), mortalidade (M), brotações (B), número de raízes (NR), comprimento das três maiores raízes (CMTR) e número de brotações (NB) (Tabela 1).

Para a variável porcentagem de enraizamento (E), constatou-se diferença significativa entre as estacas sem folhas $(57,50 \%)$ e as com folhas (um par e dois pares), ambos com 95,00\% de enraizamento (Tabela 1).

Tabela 1. Dados médios para as variáveis porcentagem de enraizamento (E), mortalidade (M), brotações (B), número de raízes (NR), número de brotações (NB) e comprimento das três maiores raízes (CMTR), sob diferentes tipos de estacas (TE), no enraizamento de Sanchezia oblonga. UTFPR, Dois Vizinhos, Paraná, 2020.

\begin{tabular}{|c|c|c|c|c|c|c|}
\hline \multirow{2}{*}{ TE } & E & $\mathbf{M}^{(\mathrm{t})}$ & B & \multirow{2}{*}{ NR } & \multirow{2}{*}{ NB } & CMTR \\
\hline & \multicolumn{3}{|c|}{. } & & & $\mathrm{Cm}$ \\
\hline Sem folhas & $57,50 b^{*}$ & $42,50 \mathrm{a}$ & $57,50 \mathrm{~b}$ & $5,85 \mathrm{c}$ & $2,30 \mathrm{a}$ & $7,77 \mathrm{~b}$ \\
\hline 1 Par & $95,00 \mathrm{a}$ & $2,50 \mathrm{~b}$ & $87,50 \mathrm{ab}$ & $8,87 \mathrm{~b}$ & $1,64 \mathrm{~b}$ & $10,10 \mathrm{a}$ \\
\hline 2 Pares & $95,00 \mathrm{a}$ & $5,00 \mathrm{~b}$ & $97,50 \mathrm{a}$ & $13,45 \mathrm{a}$ & $1,53 \mathrm{~b}$ & $10,42 \mathrm{a}$ \\
\hline Média & 82,50 & 16,66 & 80,83 & 9,39 & 1,82 & 9,40 \\
\hline CV \% & 19,27 & 40,87 & 19,95 & 15,34 & 9,98 & 20,85 \\
\hline DMS & 31,41 & 31,24 & 31,06 & 2,84 & 0,36 & 2,25 \\
\hline
\end{tabular}

CV - Coeficiente de variação. DMS - Diferença mínima significativa. ${ }^{(t)}$ Transformado por arcseno da $(\sqrt{ } x+5)$. *As medias seguidas com mesma letra não diferem significativamente a $5 \%$ pelo teste Tukey. Fonte: Autores (2020).

Percebeu-se que as folhas presentes nas estacas de S. oblonga influenciaram positivamente no sucesso do enraizamento. Esse fato pode ser explicado por Paiva e Gomes (2011) que ressaltam que a presença foliar nas estacas é importante, pois nelas são realizados os processos fotossintéticos. Segundo Taiz, et al. (2017) durante a fotossíntese, são produzidos fotoassimilados como os carboidratos que servem de suporte metabólico para produção de energia e promovem a formação e crescimento de raízes.

Moraes, et al. (2016), estudando a propagação de pingo-de-ouro via estaquia, verificaram que a ausência de folhas inviabilizou o enraizamento das estacas e que o número ideal de folhas para enraizamento desta espécie é de um a dois pares de folhas inteiras. Esse mesmo autor, ressalta que a viabilidade das estacas é comprometida pela presença e ausência de folhas. Para Belniaki, et al. (2018) a área foliar mantida em estacas caulinares tem efeito expressivo na qualidade do enraizamento.

Para a variável mortalidade (M) foi observada maior mortalidade em estacas sem folhas (42,50\%), diferindo significativamente dos tratamentos com folhas. Esse aspecto foi observado também por Pacheco e Franco (2008) no enraizamento de acoita-cavalo (Luehea divaricata), no qual 100\% das estacas sem folha morreram antes mesmo de enraizar.

Quanto a porcentagem de estacas com brotações (B) houve diferença estatística entre estacas sem folhas $(57,50 \%)$ e com dois pares de folhas $(97,50 \%)$ (Tabela 1). Moraes, et al. (2016) também obtiveram índice mais elevado de brotações nas 
estacas que apresentaram de um a dois pares de folhas. Para Santos, et al. (2017) a presença de folhas aumentou a brotação de murta (Murraya exótica). Ressaltaram ainda que a produção de fotoassimilados e de moléculas com ação hormonal nas folhas mantidas nas estacas podem ser significativas para elevada brotação.

Já para número de brotações (NB) o tratamento estacas sem folhas $(2,30)$ foi superior no estímulo a formação de brotações. Esse fato pode estar relacionado com o direcionamento da reserva energética das estacas (Hartmann, et al., 2018). Com a retirada das folhas das estacas, formaram-se locais de abertura, podendo a reserva enérgica armazenada (carboidratos) na estaca ir em direção a esses locais cicatrizando-os, ou até mesmo aos locais estejam mais próximos ao de reserva energética, provendo a diferenciação e formação de um maior número brotações (Taiz, et al., 2017; Bhatla; Lal, 2018). Outro aspecto a ser considerado, é devido às folhas serem fontes de auxina, que é transportada em direção à base da estaca, ocasionando assim a inibição do desenvolvimento das gemas laterais (Sousa, et al., 2014; Santos; Marques; Sousa, 2017).

Em relação as variáveis número de raízes (NR) e comprimento médio de raízes foi constatada diferença estatística entre os tratamentos, onde para NR, estacas com dois pares de folhas foram significativamente superior $(13,45)$ aos demais tratamentos no estímulo à formação de raízes. Já para CMTR estacas com presença de folhas estimularam a formação de raízes, sendo até $34 \%$ mais longas que em estacas sem folhas (Tabela 1). As folhas atuam como fonte de carboidratos, água, hormônios, dentre outros cofatores (compostos nitrogenados, aminoácidos, compostos fenólicos e outras substâncias não identificadas) importantes para formação e crescimento das raízes (Paiva; Gomes, 2011; Santos; Marques; Sousa, 2017; Hartmann, et al., 2018).

As folhas possuem função importantíssima na viabilidade das estacas, pois atuam como principal fonte de carbono que auxilia na realização da fotossíntese, sendo a estrutura responsável por fornecer os suprimentos necessários para o processo de rizogênese e garantir o desenvolvimento das raízes (Santos; Marques; Sousa, 2017; Belniaki, et al., 2018)

A sobrevivência (S) foi baixa devido ao elevado índice de enraizamento em estacas com folhas, não havendo diferença significativa entre os tratamentos, e também à elevada mortalidade (47,50\%) em estacas sem folhas (Tabela 2).

Tabela 2. Dados médios para a variável sobrevivência (S), sob diferentes tipos de estacas (TE) no enraizamento de Sanchezia oblonga. UTFPR, Dois Vizinhos, Paraná, 2020.

\begin{tabular}{cc}
\hline TE $(\mathrm{cm})$ & $\mathrm{S}(\%)$ \\
\hline Sem folhas & $0,00 \mathrm{a}^{*}$ \\
1 Par & $2,50 \mathrm{a}$ \\
2 Pares & $0,00 \mathrm{a}$ \\
\hline Média & 0,83 \\
\hline CV \% & 36,41 \\
DMS & 5,70 \\
\hline
\end{tabular}

CV - Coeficiente de variação. DMS - Diferença mínima significativa. *As medias seguidas com mesma letra na coluna não diferem significativamente a 5\% pelo teste Tukey. Fonte: Autores (2020).

Os resultados deste trabalho indicam que a presença foliar nas estacas de $S$. oblonga influenciou positivamente a produção de mudas desta espécie. De acordo com estudos de Moraes, et al. (2014) a redução em 50\% da área foliar de estacas de eucalipto promove o pegamento das mudas. Batista, et al. (2014) verificou que a presença de folhas em miniestacas de eucalipto, influenciou a sobrevivência e enraizamento, com melhores resultados em folhas inteiras e reduzidas à metade. Nogueira, et al. (2017) obteve sucesso na propagação via estaquia de Ficus benjamina em estacas com um par de folhas 
reduzidas à metade. Masiero, et al. (2021) relatou que a presença de folhas em estacas de melissa (M. oficinais) influenciou no enraizamento e na formação de brotações.

\section{Experimento 2}

No segundo experimento, para o comprimento das estacas, constatou-se diferença significativa apenas para a variável número de brotações (NB), sendo que as estacas de maiores comprimentos, ou seja, com 12 e $18 \mathrm{~cm}$ apresentaram número de brotaçoes superior ao obtido em estacas de de $6 \mathrm{~cm}$ (Tabela 3). Pizzatto, et al. (2011), estudando a propagação de hibisco (Hibiscus rosa-sinensis) via estaquia, verificaram em estacas com $12 \mathrm{~cm}$ de comprimento resultados superiores para as variáveis porcentagem de enraizamento e número de brotações, quando comparadas às estacas de $6 \mathrm{~cm}$.

Os autores supra citados, ainda relataram que o tamanho da estaca influencia na quantidade de reserva nutricional. Quanto maior o comprimento das mesmas, maior será o conteúdo de carboidrato de reserva, consequentemente maior a utilização no processo de enraizamento e brotação (Souza, et al., 2012).

Tabela 3. Dados médios para a variável número de brotações (NB) sob diferentes comprimentos de estacas (CE), no enraizamento de Sanchezia oblonga. UTFPR, Dois Vizinhos, Paraná, 2020.

\begin{tabular}{cc}
\hline $\mathrm{CE}(\mathrm{cm})$ & $\mathrm{NB}$ \\
\hline 6 & $1,84 \mathrm{~b}^{*}$ \\
12 & $2,61 \mathrm{a}$ \\
18 & $2,62 \mathrm{a}$ \\
\hline Média & 2,35 \\
\hline CV \% & 10,72 \\
DMS & 0,53 \\
\hline
\end{tabular}

CV - Coeficiente de variação. DMS - Diferença mínima significativa. *As medias seguidas com mesma letra não diferem significativamente a 5\% pelo teste Tukey. Fonte: Autores (2020).

Com relação as variáveis de enraizamento (E), mortalidade (M), brotações (B), número de raízes (NR) e comprimento das três maiores raízes (CMTR), os tratamentos não diferiram estatisticamente. Assim, para as variáveis E e B foram observados altos índices de desenvolvimento, com médias próximas a 100\% para ambas, respectivamente. Devido ao sucesso no enraizamento houve baixa mortalidade de estacas (3,33\%). Em relação a variável NR, verificou-se média geral de 13,86 raízes por estaca, enquanto que para o CMR, observou-se média de 10,69 cm (Tabela 4). 
Tabela 4. Dados médios para as variáveis porcentagem de enraizamento (E), mortalidade (M), brotações (B) e para número de raízes (NR) e comprimento das três maiores raízes (CMTR) por estaca, sob diferentes comprimentos de estacas (CE), no enraizamento de Sanchezia oblonga. UTFPR, Dois Vizinhos, Paraná, 2020.

\begin{tabular}{|c|c|c|c|c|c|}
\hline \multirow{2}{*}{$\mathrm{CE}(\mathrm{cm})$} & $\mathrm{E}$ & M & B & \multirow{2}{*}{ NR } & \multirow{2}{*}{$\begin{array}{l}\text { CMR } \\
(\mathrm{cm})\end{array}$} \\
\hline & \multicolumn{3}{|c|}{ 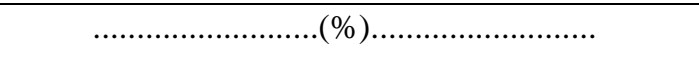 } & & \\
\hline 6 & $100,00 \mathrm{a}^{*}$ & $0,00 \mathrm{a}$ & $95,00 \mathrm{a}$ & $12,75 \mathrm{a}$ & $13,02 \mathrm{a}$ \\
\hline 12 & $100,00 \mathrm{a}$ & $0,00 \mathrm{a}$ & $100,00 \mathrm{a}$ & $14,60 \mathrm{a}$ & $10,06 \mathrm{a}$ \\
\hline 18 & 96,60 a & $3,33 \mathrm{a}$ & $96,60 \mathrm{a}$ & $14,36 \mathrm{a}$ & 8,99 a \\
\hline Média & 98,86 & 1,11 & 97,27 & 13,86 & 10,69 \\
\hline $\mathrm{CV} \%$ & 2,91 & 31,7 & 4,69 & 11,87 & 17,49 \\
\hline DMS & 6,15 & 6,15 & 9,73 & 3,35 & 4,04 \\
\hline
\end{tabular}

CV - Coeficiente de variação. DMS - Diferença mínima significativa. *As medias seguidas com mesma letra não diferem significativamente a $5 \%$ pelo teste Tukey. Fonte: Autoria própria (2020).

Por meio dos resultados obtidos pode-se constatar que a espécie é facilmente propagada pela técnica de estaquia, ou seja, de fácil enraizamento. No estudo realizado por Weiss, et al. (2018), com estacas de Sanchezia oblonga em diferentes substratos, também foram obtidos elevados índices de enraizamento, com média geral de 83,33\%. Os resultados obtidos podem ter relação com a utilização do substrato vermiculita, por apresentar boa aeração, drenagem e alta capacidade de retenção hídrica (Hartmann, et al., 2018).

\section{Conclusão}

Sanquésia é facilmente propagada pela técnica de estaquia, sendo considerada uma espécie de fácil enraizamento. Para o sucesso na produção de mudas dessa espécie podem ser utilizadas estacas de qualquer comprimento, com presença de um ou dois pares de folhas reduzidos à metade.

Com este estudo é possível compreender aspectos da propagação vegetativa da sanquésia, para sua utilização na ornamentação. Diante disso, reforçamos a necessidade de novos estudos sobre a propagação vegetativa da espécie, como forma de realizar utilização em projetos ornamentais.

\section{Referências}

Alvares, C. A., Stape, J. L., Sentelhas, P. C., Onçalves, J. L. M., \& Sparovek, G. (2013). Köppen’s climate classification map for Brazil. Meteorologische Zeitschrift, 22 (6), 711-728.

Batista, A. F., Santos, G. A., Silva, L. D., Quevedo, F. F., \& Assis, T. F. (2014). Influência da arquitetura foliar de miniestacas na propagação clonal de Eucalyptus. Revista Árvore, 38 (5), 819-827.

Belniaki, A. C., Rabel, L. A. N., Gomes, E. M., \& Zuffellato-Ribas, K. C. (2018). Does the presence of leaves on coleus stem cuttings influence their rooting?. Ornamental Horticulture, 24 (3), 206-210.

Bhatla, S. C., \& Lal, M. A. (2018). Plant Physiology, Development and Metabolism. Singapore: Springer Nature.

Costa, V. A., Jorg amici, M. H., Costa, E., Castro, A. R. R., \& Costa, M. L. N. (2016). Efeito de cortes de estacas e da presença de folhas na produção de mudas de Mentha sp. Revista Brasileira de Biociências, 14 (2), 55-59.

Espindola, A. E., \& Orenha, C. E. (2007). Flora Apícola em Santa Catarina: I - Astrapéia. Informativo Zum-Zum. 330. 
Fachinello, J. C., Hoffmann, A., \& Nachtigal, J. C. (2005). Propagação de plantas frutíferas. UFPEL, 2005.221 p.

Ferreira, D. F. (2011). SISVAR: a computer statistical analysis system. Ciência e Agrotecnologia, 35 (6), 1039-1042.

Hartmann, H. T., Kester, D. E., Davies Jr, F. T., Geneve, R. L., \& Wilson, S. E. (2018). Plant propagation: principles and practices. New Jersey: Prentice Hall.

Instituto Nacional de Meteorologia do Brasil - INMET. Normais Climatológicas de Dois Vizinhos (2016). http://www.inmet.gov.br/portal/index.php?r=estacoes/estacoesAutomaticas.

Lorenzi, H., \& Souza, H. M. (2018). Plantas ornamentais no Brasil: arbustivas, herbáceas e trapadeiras. Nova Odessa: Instituto Plantarum.

Marchi, P. M., Antunes, L. E. C., Pereira, I, S., Höhn, D., \& Valgas, R. A. (2018). Vegetative propagation of raspberry from leafy cuttings. Revista Brasileira de Fruticultura, 40 (5), (e-378).

Masiero, M. A., Viana, C. M. S. S., Lupepsa, C. T., Silva, F. R., Almeida, G. M. C., Tombolato, J. P., Carolino, K., Silva, R. Q., \& Lima, D. M. (2021). Propagação vegetativa de Melissa officinalis L. por estaquia. Biodiversidade, 20 (1), 122-128.

Moraes, C. E., Fonseca, R. C. M., \& Rui, M. (2014). Influência das folhas no enraizamento de miniestacas de híbridos de eucalipto. Nucleus, 11 (1), 101-106.

Moraes, E. R., Santos, M. S., Peixoto, J. V., \& Golinski, J, (2016). Produção de mudas de pingo-de-ouro sob diferentes tamanhos de estacas e quantidades de folhas. Enciclopédia Biosfera, 13 (23), 1063.

Nogueira, G. S., Silva, F. A. C., Kunze, G., Figueiró, J. P. S., Kruchelski, S., \& Ribas, K. C. Z. (2017). Influência do número de folhas e da aplicação de IBA na estaquia caulinar de Ficus benjamina L. Revista Agrarian, 10 (36), 113-119.

Paiva, H. N., \& Gomes, J. M. (2011). Propagação vegetativa de espécies florestais. UFV.

Pacheco, J. P., \& Franco, E. T. H. (2008). Substratos e estacas com e sem folhas no enraizamento de Luehea divaricata Mart. Ciência Rural, 38 (7), 19001906.

Pereira, A. S., Shitsuka, D. M., Pereira, F. J., \& Shitsuka, R. (2018). Metodologia da pesquisa científica. UFSM,.

Pizzatto, M., Wagner Júnior, A., Luckmann, D., Pirola, K., Cassol, D. A., \& Mazaro, S. M. (2011). Influência do uso de AIB, época de coleta e tamanho de estaca na propagação vegetativa de hibisco por estaquia. Revista Ceres, 58 (4), 487-492.

Santos, M. P., Marques, R. C., \& Sousa, C. M. (2017). Enraizamento de estacas caulinares de murta (Murraya exotica L.). Scientific Electronic Archives, 10 (2), 5-10.

Souza, E. R., Ribeiro, V. G., Mendonça, O. R., Santos, A. S., \& Santos, M. A. C. (2012). Comprimentos de estacas e AIB na formação de porta-enxertos de videira 'Harmony' e ‘Campinas'. Revista Brasileira de Tecnologia Aplicada nas Ciências Agrárias, 5 (2), 19-32.

Sousa, C. M., Santos, M. P., \& Carvalho, B. M. Enraizamento de estacas de maracujazeiro-doce (Passiflora alata Curtis). Científica, 42, 68-73.

Souza, R. R., Cavalcante, M. Z. B., Lima, M. P. D., Alixandre, T. F., \& Nascimento, R. T. (2015). Propagação vegetativa de hibisco com diferentes tipos de estacas e concentrações de ácido indolbutírico. Comunicata Scientiae, 6 (3), 291-296.

Taiz, L., Zeiger, E., Møller, I. A., \& Murphy, A. (2017). Fisiologia e desenvolvimento vegetal. Artmed.

Tavares, I. B., Momnté, V. G., Barreto, H. G., Castro, H. G., Santos, G. R., \& Nascimento, I. R. (2012). Tipos de estacas e diferentes substratos na propagação vegetativa de erva cidreira (QUIMIOTIPOS I, II E III). Bioscience Journal, 28 (2), 206-213.

Weiss, E. C., Pilonetto, D., Antunes, R., Masiero, M. A., \& Lima, D. M. (2018). Estaquia de Sanchezia oblonga com a utilização de diferentes substratos. Natureza online, 16 (3), 001-007.

Xavier, A., Wendling, I., \& Silva, R. L. (2013). Silvicultura clonal: princípios e técnicas. UFV. 